\title{
Assessment of the Early Infant Diagnosis of HIV Infection in Togo in 2014

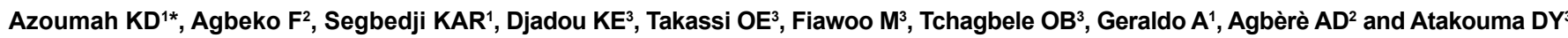 ${ }^{1}$ Department of Paediatrics, CHU-Kara, University of Kara, Kara, Togo \\ ${ }^{2}$ Department of Paediatrics, CHR Lomé Commune, University of Lomé, Lomé, Togo \\ ${ }^{3}$ Department of Paediatrics, CHU-Sylvanus Olympio, University of Lomé, Lomé, Togo
}

\begin{abstract}
Introduction: Early infant diagnosis (EID) of Human immunodeficiency virus (HIV) infection by Polymerase Chain Reaction (PCR) on Dry Blood Spot (DBS) has started in Togo since 2009 with difficulties about geographic accessibility and durability of the test. The aim of this study is to analyze the timeframes of implementation and the results of the PCR in Togo.

Methodology: This was a cross-sectional study from 22 July to 15 August 2014 on PCR registers and application forms in the two reference laboratories in Togo (Lomé and Kara) and on clinical records of exposed children in 26 sites of Prevention of mother-to-child transmission (PMTCT). The analysis focused on the sampling, performance and test results.

Results: The number of collected samples increased from 450 in 2011 to 920 in 2013, representing only $13 \%$ of the expected PCRs. However, $97 \%$ of these samples were sent to laboratories. The average age at PCR samples varied from 3.3 to 8.5 months. The main gateway was PMTCT $(99.5 \%)$; the majority of children were breastfed $(90.8 \%)$. The proportion of results returned to parents ranged from $12 \%$ to $52 \%$ between 2011 and 2013 . The average duration between the dates of the sample's withdrawal and the return of the result to the mother, calculated on clinical record, was 68 days $(n=62$ ) but could reach 105.5 days according to the health service providers. The rate of vertical transmission of HIV was $4.5 \%$.
\end{abstract}

Conclusion: The timeframes for the implementation of PCRs are long in Togo. Scaling up and creating an emergency fund will improve the early diagnosis of the child.

Keywords: PCR; Early infant diagnosis; HIV; Togo

\section{Introduction}

Human immunodeficiency virus (HIV) infection remains a public health problem in resource-limited countries, 91\% of HIV-infected children in the world live in sub-Saharan Africa [1]. The prevalence of HIV was $2.5 \%$ in 2013 in Togo. Togo is one West African small country with a population of $6,600,000$ and a ratio of one doctor per 16,670 inhabitants. HIV infected-children under fifteen were estimated 21,000 making up $7 \%$ of the active list. The third of these children die before 12 months and the half $(50 \%)$ before 2 years [1,2]. Despite advances in prevention of mother-to-child transmission (PMTCT) and management, the diagnosis of HIV's infection in children remains delayed in Togo, around 5 years old children [3]. Like the global recommendations for Early Infant Diagnosis (EID) of HIV, Togo retained a first Polymerase Chain Reaction (PCR) for a six-week visit and a second, for the breastfed child, at six weeks after discontinuing any exposure to breast milk. HIV serology was performed in 12-month-old children with confirmation at 18 and 24 months when the 12 -month test was positive [3-5]. The mother-baby couple was followed for at least two years in one of the following services: maternity, pediatrics or medicine. EID of HIV at six weeks has been started in Togo since 2009 by PCR on Dry Blood Spot (DBS). However, poor geographic accessibility and regular breaks in the supply of inputs hamper the performance of this test. The aim of this study is to analyze the different conditions and the results of the PCR in Togo in order to propose strategies to overcome difficulties that impede better performance.

\section{Methodology}

This was a cross-sectional descriptive study from July 22 to August 15,2014 of a quantitative and qualitative mixed design data collection after prior agreement of the national ethics committee. It covered PCR registers and application forms in the two reference laboratories in Togo
(Lomé and Kara) as well as the clinical records of the children exposed, randomly selected at 26 PMTCT sites. This review was supplemented by an interview with PMTCT site staff.

The choice of the chosen health services took into account the health pyramid, the type of institution (public, associative, confessional or lucrative), the rural or urban character and the active list of HIVexposed children being observed. The institutions selected at the central level for the situation analysis include the two national reference laboratories for early diagnosis (Lomé for the southern part of the country and Kara for the whole northern part) and two of the three reference centers in Togo: Centre Hospitalier Universitaire (CHU) Sylvanus Olympio in Lomé and CHU Kara. At the intermediate level, a careful choice based on more than one year of experience in the field of pediatric HIV care and the availability of a pediatric active list of more than $5 \%$ of the national list has led us to retain the Centre Regional Hospital of Tsévié. At the peripheral level, six districts were selected (one district per health region) on the basis of a reasoned choice based on the size of the active list of HIV infected-children and the absence of a Regional Hospital in the area of the district. In selected districts, in addition to the district hospital, two peripheral health units (Type I or

*Corresponding author: Azoumah KD, Head of department, Universite de Kara/ CHU-Kara (Kara Teaching Hospital), Paediatrics, Boulevard des Evala, Kara, Togo, Tel: 0022890019866; E-mail: adkazoum@gmail.com

Received May 02, 2017; Accepted May 18, 2017; Published May 25, 2017

Citation: Azoumah KD, Agbeko F, Segbedji KAR, Djadou KE, Takassi OE, et al (2017) Assessment of the Early Infant Diagnosis of HIV Infection in Togo in 2014. J AIDS Clin Res 8: 695. doi: 10.4172/2155-6113.1000695

Copyright: (C) 2017 Azoumah KD, et al. This is an open-access article distributed under the terms of the Creative Commons Attribution License, which permits unrestricted use, distribution, and reproduction in any medium, provided the original author and source are credited. 
Type II) providing PMTCT services were selected, including a PMTCT site collecting DBS for EID and another PMTCT site not doing it. One of these health facilities was selected in rural areas while the other is urban. Both sites must have delivered at least one HIV-infected woman during the year 2013. The confessional health units representing $11 \%$ of the national active list of HIV infected-children were included at the rate of one organisation by each southern and northern region of Togo. As for the associative organisations involved in the management of HIV (about $40 \%$ of HIV-infected children in Togo), two were selected in Lomé and one in the North of Togo; as well as a private health facility. Interviews were conducted with health care providers in the various departments and key informants who are program managers at central level, as well as technical and financial partners. The surveyed health service providers are those generally involved in pre-natal consultation and/or maternity, PMTCT, blood collection on DBS, post-natal or curative counseling, immunization or nutrition.

Data collection was provided by a team of twelve (12) trained health professionals (4 paediatricians, 2 general practitioners, 5 senior health technicians and one statistician). Five tools consisting of interview guides and data mining sheets were used after pretexting: Tool A (PMTCT and HIV/AIDS pediatric data collection); Tool B (semistructured interview guide with managers), Tool C (semi-structured interview guide with providers), Tool D (on-site data extraction sheets) and Tool E (reference laboratory record). The quantitative analysis included infant registries, feeding mode, PCR delivery portal, number of PCRs performed, dates of collection and reporting of PCR results. The qualitative treatment was manual, consisting of a triangulation of the results so as to cross the information from different sources and concerns the level of coverage, accessibility and availability of PCR services, difficulties and challenges.

\section{Results}

\section{Overall results}

The number of health facilities offering PMTCT increased from 411 in 2012 to 596 in 2013 (of which 554 were public health facilities). The PMTCT service providers increased from $47.56 \%$ to $87.66 \%$ and PCR from $16.1 \%$ to $27.7 \%$. Of all exposed children expected, $89 \%$ were seen at vaccinating Penta 1 at six weeks. This percentage was $19 \%$ in postnatal consultation and 13\% in PCR; however, 97\% of the PCR samples were sent to national laboratories.

\section{Overall description of the early infant diagnosis circuit in Togo}

The overall organisation of the early diagnosis circuit in Togo is provided by the PNLS, which has contracted with the national postal service to forward samples to the national reference laboratory BIOLIM Lomé for early diagnosis. The creation of a second laboratory in 2013, BIOLIM Kara in the north of Togo has allowed an extension of the geographical coverage of sampling sites. The staff of the national early diagnostic laboratory of BIOLIM Lomé consisted of two virologists and two part-time laboratory engineers. The Kara laboratory is run by a part-time senior laboratory technician supported by a medical biologist coming on special duty from Lomé. Average hours of work were six hours per day per person. BIOLIM Lomé processed about 100 samples per week with a maximum capacity of 160 . In Kara the average was 2.5 samples per week. The average number of samples received at BIOLIM Lomé is 220 per month, compared with 10 at BIOLIM Kara for the period from May to July 2014.

The DBS samples are conveyed by the National Post service to the national laboratory BIOLIM Lomé. Some sampling sites send their samples BIOLIM Lomé by their own means. BIOLIM Kara, the laboratory in the north of Togo, has not yet been formalized in the contract with the post, but the samples from the three northern regions pass through BIOLIM Lomé before reaching it. The results were returned to the healthcare facilities also by postal mail. These results were prepared under sealed envelopes, the discharge booklet signed by the postal officer. Some centres in Lomé picked directly their results at BIOLIM Lomé. There was an internal and external quality control inscribed on the evaluation check, with affiliation to the Center of Diseases Control (CDC) Atlanta. Between November 2013 and April 2014, breaches of the test were observed in Lomé for 180 days and in Kara for 14 days, due to a delay in funding linked to the procedures of the Global Fund.

\section{Procedure for sampling and transport of samples}

Seventeen of the 26 centres performed early diagnosis. This service was almost exclusively available in public health facilities (15/17). Blood collection by a trained agent from the centre was the most common sampling procedure $(17 / 17)$. The direct reference to the national laboratory $(4 / 17)$, the collection of grouped blood by a trained agent from another centre (1/17) and the reference to a sampling centre $(1 / 17)$ were also cited. DBS sampling sessions were generally weekly on sites with schedule for sending and receiving samples (12/17), except at the CHU Sylvanus Olympio (once every 2 weeks). In case of delay or absence of sample, the sampling agent use to contact the Post Office directly (3/17). Some No Government Organizations (NGOs) or confessional hospitals also took the initiative to gather DBS samples and send them to the reference collection centre (the Post Office is not affiliated to all institutions). The movement of mothers to the collection centres through support for transport was sometime facilitated by some NGOs.

BIOLIM receives the samples on Tuesdays and Thursdays from the Post Office. The results were sent by the same way to the sending institutions. These results are distributed to patients' centres of origin at the monthly coordination meetings held in the districts and by the focal points. Rejected or invalid samples were re-sampled after informing the providers by telephone and written. Then a copy of the completed PCR application form was returned to the site.

\section{Average DBS circuit stage delays}

During the interviews with health care providers, the average time between collection and shipment of DBS samples was 5.5 days, between the sending and receiving of the result by the health worker the time was 74 days and from the latter to the mother of the child, 26 days. The total duration was around 105.5 days. The second stage was longer in rural areas, 100 days compared with 71 days in urban areas. For the third stage, urban services reported their results later (30 days) than the rural ones (14 days). Table 1 details the estimated time and distance between health facilities and the Lomé laboratory.

The total duration, from the date of sampling and rendering of results, calculated on clinical record was 68 days $(n=62)$. Table 2 shows the different delays in the early diagnosis circuit based on data from the site: these delays overall are long.

\section{Community-based services for early detection and pediatric treatment}

Community services were generally underdeveloped in public training in Togo, especially in PMTCT and pediatric HIV. The main 
Citation: Azoumah KD, Agbeko F, Segbedji KAR, Djadou KE, Takassi OE, et al. (2017) Assessment of the Early Infant Diagnosis of HIV Infection in Togo in 2014. J AIDS Clin Res 8: 695. doi: 10.4172/2155-6113.1000695

Page 3 of 5

\begin{tabular}{|l|c|c|c|c|c|c|}
\hline & \multicolumn{2}{|c|}{ Place of résidence } & \multicolumn{4}{c|}{ Health level } \\
\hline & Urban & Rural & Central & Intermediate & Peripheral 1 & Peripheral 2 \\
\hline Average Time $\mathbf{( m i n )}$ & 209 & 283 & 13 & 40 & 174 & 346 \\
\hline Average Distance $\mathbf{( k m )}$ & 125 & 193 & 2 & 25 & 143 & 190 \\
\hline
\end{tabular}

Table 1: Estimated time and distance by road from the site to the reference laboratory.

\begin{tabular}{|c|c|c|c|c|}
\hline Duration (Days) & Number of files & Minimum & Maximum & Average \\
\hline Duration between the beginning of Prophylaxis or the triple therapy of the mother and the birth of the child & 36 & 0 & 79 & 19 \\
\hline Duration between the birth of the child and the prophylaxis & 69 & 0 & 71 & 3 \\
\hline Duration between the birth of the child and the first sampling & 73 & 0 & 476 & 88 \\
\hline Duration between first sampling and sending to the laboratory & 63 & 0 & 27 & 6 \\
\hline Duration between sending of the first sample and arrival at the reference laboratory & 47 & 0 & 72 & 11 \\
\hline Duration between arrival at the laboratory and delivery of the result of sampling to the health facility & 48 & 5 & 186 & 59 \\
\hline Duration between the first sample and receipt of the result to the Institution & 62 & 12 & 189 & 68 \\
\hline Duration between the receipt of the result by the Institution and the delivery of the result to the mother & ND & ND & ND & ND \\
\hline Duration between delivery of result to mother and HAART & ND & ND & ND & ND \\
\hline
\end{tabular}

ND: Not Determined; HAART: High Active Antiretroviral Therapy

Table 2: Different delays in the cascade of early HIV diagnosis.

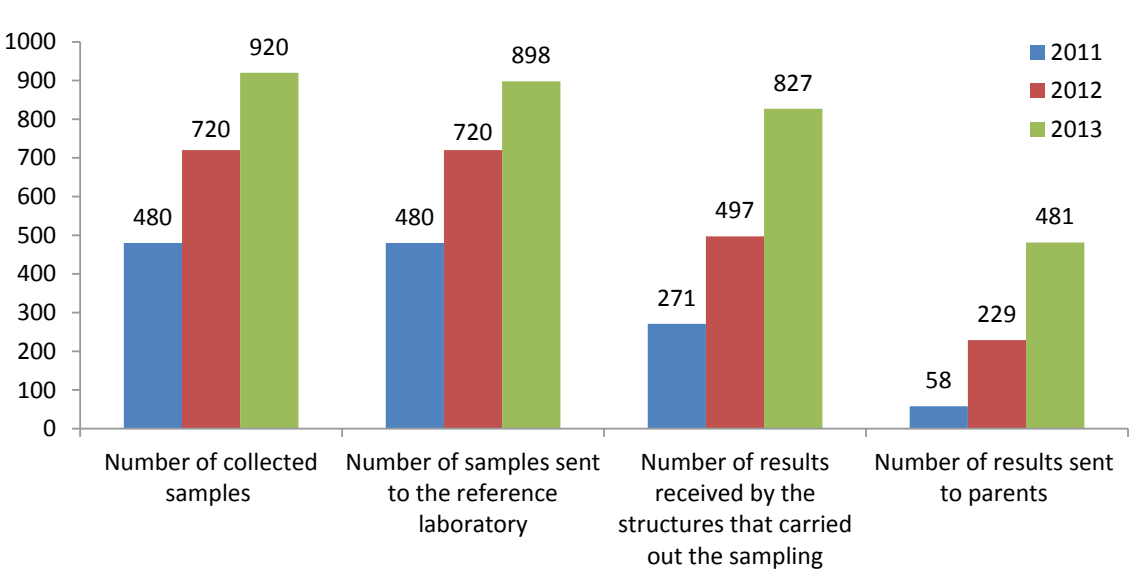

Figure 1: Cascade of the number of DBS samples from the sampling to the result of the parents.

reasons were the lack of financial resources associated with insufficient social mediators. However, these psychosocial agents acted effectively where they were posted, by searching for untrained or lost-to-follow infants. The current status of these social mediators is undetermined but is being formalized.

\section{Results of early diagnosis in the centers visited}

There was an increase from 450 to 920 samples recorded from 2011 to 2013 (51.1\%). Similarly, the proportion of results returned to parents improved from $12 \%$ to $52 \%$ over the same period. The average age of children tested in the last months of 2014 in Lomé and Kara respectively was: in May 8.5 months and 3.3 months, in June 8.2 months and 4.6 months and in July of 6.3 months and 4.2 months. The feeding pattern of these children was predominantly breastfeeding $(90.8 \%)$. The number of indeterminate results in the last 12 months was $0 \%$ in Lomé and $1.9 \%$ in Kara. Recoveries were all negative. The rate of mother-tochild transmission (MTCT) of HIV after EID was 4.5\%. Figure 1 shows the cascade of the number of collected samples from the sampling to the delivery of results to the parents per year.

At the end of the interview with the users in the organisation of the early diagnosis, certain constraints were noted: absence or delay of rendering of results (lack of reagents), circuit with the post unclear for
BIOLIM Kara, patients failure to fulfill medical visits, stigmatization in health facilities, inadequate human resources, lack of certain key information on collection tools, irregular supervision, absence of a framework for exchanges between clinicians and laboratory staff, inadequate or cramped premises, lack of energy autonomy, insufficient maintenance.

\section{Discussion}

\section{Why early diagnosis}

The poor retention of mother-baby couples in HIV care remains a challenge for the elimination of mother-to-child transmission (e-MTCT) from HIV, hence the need for early infant diagnosis (EID). In Togo in 2011, 157 HIV-exposed children (34.5\%) were considered lost to follow-up prior to PCR-1 in a study on the accessibility of EID at Lomé, another 100 (21.9\%) were also loss between the completion of PCR-1 and the results of this review, an overall loss of 257 (56.6\%) [5]. In Uganda, nearly one third of mother-baby pairs are not retained in HIV care: lack of EID services, poor quality service, non-disclosure of mother's HIV status and understanding the importance of adhering to all appointments together with the baby, were associated with time to non-retention [6]. EID is one of the strategies to achieve UNAIDS (Joint United Nations Programme on HIV/AIDS) goals 90-90-90 (by 
2020, $90 \%$ of all people living with HIV will know their status, $90 \%$ of all people diagnosed with HIV will receive a supported antiretroviral therapy (ART) and 90\% of all people receiving ART will have a lasting viral suppression); however, country data for this indicator are fragmented or non-existent [7-10]. Infants infected with HIV during pregnancy, childbirth or premature deliveries often die before their HIV status is known. States should ensure that appropriate systems and tools are in place to manage supplies at all levels for EID [7,11]. Togo, with a surface area of $56600 \mathrm{Km}^{2}$ spreading over $634 \mathrm{~km}$ in length has set up a system of EID based on the different institutions and two PCR laboratories and has a plan for the elimination of mother-to-child HIV transmission (e-MTCT) by the end of 2018, which is closely aligned with the global goals and commitments. This plan should reduce the rate of vertical transmission of HIV below $5 \%$, new pediatric infections by $90 \%$ and the number of maternal deaths associated with AIDS by $50 \%[12]$.

\section{What early diagnosis}

Adding HIV testing at birth has the potential to improve the number and timing of ART initiation of HIV-infected infants ( $543 \%$ by age 3 months, 204\% by age 18 months), leading to a decrease in infant mortality. Using this model, countries should investigate a combination of HIV testing at birth and during the early infant period [13]. But what tests for EID? Early diagnosis is carried out by virological tests: qualitative or quantitative PCR with desoxyribonucleic acid (DNA) or ribonucleic acid (RNA), p24 antigen. In Togo, quantitative RNAPCR is the technique performed by the two BIOLIM laboratories. In Equatorial Guinea, the HIV-1 genome is detected using the Siemens DNA VERSANT HIV-1 RNA $1.0 \mathrm{k}-\mathrm{PCR}$ assay after making DBS [14].

Although PCR and EID achieved an average of $40 \%$ in 2013, better than CD4 (cluster of differentiation 4), there are large variations between regions [11]. Discordant results are sometimes observed: Jagtap et al. reported a series of three cases of infants, aged four to ten months, diagnosed with pediatric HIV-1 infection using DNA PCR testing and got ART and whose serological tests at age of 18 months returned negative for HIV-1. Such seroreversion may be either due to viral suppression or false positive DNA PCR results [15]. In an evaluation of four commercial virological assays for early infant HIV-1 diagnosis using dried blood specimens, these tests were all useful for EID, although discrepant results were common [16]. These studies advocate further research to reduce false-positive results that could lead to false diagnosis and unnecessary treatment, and that caution should be exercised in interpreting the results of quantitative PCR with low viral load (VL) values and in developing guidelines for the management of cases with discordant virological findings $[15,16]$.

Rapid serological tests (Determine and Unigold) were compared in Malawi between 2008 and 2012 for EID. Starting at age 6 and 9 months, the Unigold test could be used as a screening tool in the follow up of HIV-exposed infants with specificity values of $83.7 \%$ (95\% CI: $74.4,89.9)$ and $97.7 \%$ (95\% CI: 94.6, 99.7), respectively. These authors recommend updating guidelines for use of rapid tests in young HIVexposed children that explicitly take type of test and infant age in a context where PCR is not very accessible [17]. Innovative strategies need to be implemented, particularly those related to the implementation of Point-of-Care (PoC) technology for EID [5,9,11]. Despite its lower sensitivity, the PoC test had the potential to provide test results to up to $81 \%$ more patients compared to the laboratory-based test $[9,18]$ and may be useful in peripheral care environment [18]. The DBS can be used as an affordable tool to monitor the accuracy of PoC tests and be practical for quality control programs. Its use can help countries to scale up VL testing because it is affordable and practical, not requiring cold chain logistics, such as processing, transport and storage required for plasma samples $[7,11,18]$.

\section{Organisation of the early childhood diagnosis of HIV in Togo}

EID of HIV was instituted in Togo towards the end of 2009; geographical coverage was limited to Lomé the capital and surrounding areas due to the use of venous sampling to be delivered to the laboratory in two to three hours after collection. The introduction of the DBS and the postal conveyor system has made it possible to extend this service to the whole country: from 50 pilot sites at the end of 2010, Togo has increased to 239 sites in 2013. However, HIV EID remains very low: only $13 \%$ of HIV-exposed children have been screened for HIV testing in 2014 within 2 months of birth [2,3,19]. In Togo in 2011, HIV-exposed children in Lomé who had access to EID by PCR within six months of life accounted for $52.7 \%$ [5]. A low value of the EID indicator would indicate weaknesses at the system level, including inadequate national management of HIV virology kit supply, data collection and testing.

The strengthening of the capacities of the providers on the preparation of the DBS for PCR and the pediatric management of the HIV infection has made it possible to improve the follow-up of the children and especially the early initiation of ART in infected children in Togo, even if progress is to be made $[2,3,19]$. While postal services are a good strategy covering the country because of its small size, it is imperative to improve geographical accessibility, with postal services covering only large and medium-sized urban centres. This partially inclusive coverage of sites and the imperfect definition of the DBS circuit are all limiting and delaying factors.

In the management of EID circuit, focal points were designated in the regions and prefectures as well as in the main hospitals to ensure input management and follow-up. Psychologists and social mediators, key players who can assist in therapeutic education and improve the functioning of the circuit are very insufficient or non-existent, or recruited temporally. The Project Leonardo in Italy to evaluate the impact of the disease and a model of primary health care management in patients with cardiovascular disease and diabetes, with active involvement of care managers, has allowed to obtain a significant improvement in therapeutic education and patient management with unanimous satisfaction of care providers [20]. Action by program managers, focal points, psychologists and social mediators must be strengthened in the implementation of the EID circuit in Togo for better results. The break in supply of stock of reagents, the delay of ordering stock, the inadequacy of trained personnel, the high workload, the irregularity of the national laboratory's monitoring and stigmatization, and the loss of the mother-baby couple related bottlenecks in Togo [21]. Each State should ensure that appropriate systems and tools are in place to manage supplies at the central, institutional and district levels $[7,8]$.

\section{Result of HIV early infant diagnosis}

No national data were available for rapid diagnostic tests in the EID $[7,11]$. In our study, the mean age at PCR in children varied from 3.3 to 8.5 months, the main entry point was PMTCT (99.5\%). The median age at the start of the DBS analysis was earlier in Lomé of 77 days [4], comparable to the 2.4 months observed in Equatorial Guinea [14] whereas it was 7 weeks in Congo Kinshasa [22].

The MTCT rate for HIV at EID was 4.5\%. This rate varies according to the type, place and period of study, but increasingly decreasing: $6.7 \%$ in Benin in 2010, 2.5\% in Lomé (Togo) in 2011 and 2.9\% In Equatorial Guinea in $2013[5,10,14]$. While EID is the key to early initiation of 
Citation: Azoumah KD, Agbeko F, Segbedji KAR, Djadou KE, Takassi OE, et al. (2017) Assessment of the Early Infant Diagnosis of HIV Infection in Togo in 2014. J AIDS Clin Res 8: 695. doi: 10.4172/2155-6113.1000695

Page 5 of 5

HAART and the reduction of mortality associated with HIV infection, the authors emphasize the complexity of translating the results of clinical trials and WHO guidelines into the practice, initiation of ART is often slow [14,22,23]. During 2011-2015, the total number of HIV diagnostic tests performed among HIV-exposed infants within 6 weeks after birth increased in the seven PEPFAR countries studied, however, the rate of testing for EID among HIV-exposed infants was less than $50 \%$ in five countries in 2015 [24].

The most common difficulties in accessing EID tests were those encountered in the transport of specimens, the long delay between specimen collection and receipt of results, and the limitations of supply chain management [24]. In our study, the total time between the date of collection and return of PCR results in the records was 68 days (for an average of 105,5 days, depending on health professionals), with an initial standard of 14 days. In 2011, 99 (41.2\%) children who had received PCR1 in Lomé had received the result within a median of 2.5 months (range 1-9 months) and 59 children (24.6\%) had received it before $6^{\text {th }}$ month of life [5]. Some solutions have proved successful, including the use of short message service printers, community-based interventions and support and education for mothers [24,25]. Training of health care providers, integration of laboratory services into care facilities, and delegation of tasks along the EID flow improved EID results by 6 weeks $[25,26]$.

\section{Conclusion}

Despite the progress made in the implementation of PCR in Togo, access to EID of HIV remains to be reviewed. The delays in the realization and the results of the PCR in Togo are long; the difficulties impeding the performance of the early diagnosis are numerous. The expansion and continuous improvement of EID services in our high HIV prevalence countries is necessary to both reduce the vertical transmission of HIV and to promote EID for early initiation of ARVs in the management of HIV- infected children and thus reduce mortality. The empowerment of our countries in the financing of health programs, the creation of an emergency fund, the simplification of administrative and technical procedures are essential to achieve this. In addition to continuing the training of health personnel, other innovative initiatives for substantive mobilization, delivery and reception of results, and simple early diagnosis tests should be developed.

\section{Acknowledgement}

- UNICEF (United Nations Children's Fund) Togo.

- Programme National de Lutte contre le VIH/SIDA/IST (PNLS) Togo.

\section{References}

1. UNAIDS (2013) Global Report: UNAIDS Report on the Global AIDS Epidemic. UNAIDS; Geneva, Switzerland.

2. Ministère de la santé (2014) Enquête Démographique et de Santé. Togo.

3. Ministère de la santé (2013) Rapport PNLS 2013. PNLS-IST du Togo, Lomé.

4. World Health Organization (2013) WHO recommendations on the diagnosis of HIV infection in infants and children. WHO.

5. Bouraima M, Salou M, Tchounga BK, Lawson-Evi K, Kodjovi DK, et al. (2014) Accessibilité au diagnostic précoce de l'infection à $\mathrm{VIH}$ chez les enfants à risque de Lomé (Togo). Arch Pediatr 21: 1093-1100.

6. Obai G, Mubeezi R, Makumbi F (2017) Rate and associated factors of nonretention of mother-baby pairs in HIV care in the elimination of mother-to-child transmission programme, Gulu-Uganda: A cohort study. BMC Health Serv Res 17: 48 .

7. WHO (2015) HIV diagnostic tests in low- and middle-income countries: Forecasts of global demand for 2014-2018. Technical report, WHO, Geneva.

8. ONUSIDA, OMS (2015) Rapport d'activité 2015 sur la riposte au sida dans le monde. Directives, Genèvre 236.

9. Diallo K, Modi S, Hurlston M, Beard RS, Nkengasong JN (2016) A proposed framework for the implementation of early infant diagnosis point-of-care. AIDS Res Hum Retroviruses 33: 203-210.

10. Tchiakpe E, Hounto-Ogouyemi A, Diop Ndiaye H, Diouara AA, Aïssi AK, et al (2016) Use of dried blood spots in early diagnosis of HIV-1 infection in children born to HIV-infected mothers as part of the prevention of mother-to-child transmission in Benin. Bull Soc Pathol Exot 109: 155-159.

11. WHO (2015) WHO annual meeting with diagnostic manufacturers and stakeholders: Global forecasts of diagnostic demand for 2014-2018. Technical report Geneva, Switzerland.

12. Ministère de la santé (2014) Document du plan national e-TME. PNLS-IST du Togo, Lomé.

13. Chiu A, Modi S, Rivadeneira ED, Koumans EH (2016) Optimizing infant HIV diagnosis in resource-limited settings: Modeling the impact of HIV DNA PCR testing at birth. J Acquir Immune Defic Syndr 73: 454-462.

14. Prieto-Tato LM, Vargas A, Álvarez P, Avedillo P, Nzi E, et al. (2016) Early diagnosis of human immunodeficiency virus-1 in infants: The prevention of mother-to-child transmission program in Equatorial Guinea. Enferm Infecc Microbiol Clin 34: 566-570.

15. Jagtap DD, Gawde NC, Kamble SV, Sharma AP, Paranjape RS (2016) Discordant HIV DNA PCR results among infants diagnosed with HIV infection and initiated on ART: A case series. Int J STD AIDS 28: 415-417.

16. Alvarez P, Prieto L, Martín L, Obiang J, Avedillo P, et al. (2017) Evaluation of four commercial virological assays for early infant HIV-1 diagnosis using dried blood specimens. Pediatr Res 81: 80-87.

17. Smith ER, Sheahan AD, Heyderman RS, Miller WC, Wheeler S, et al. (2016) Performance of HIV rapid tests among breastfeeding, Malawian infants. Pediatr Infect Dis J 36: 405-411.

18. Meggi B, Bollinger T, Mabunda N, Vubil A, Tobaiwa O, et al. (2017) Point-ofcare p24 infant testing for HIV may increase patient identification despite low sensitivity. PLoS ONE 12: e0169497.

19. Ministère de la santé (2012) PNDS 2012-2015. . Ministère de la santé du Togo, Lomé.

20. Ciccone MM, Aquilino A, Cortese F, Scicchitano P, Sassara M, et al. (2010) Feasibility and effectiveness of a disease and care management model in the primary health care system for patients with heart failure and diabetes (Project Leonardo). Vasc Health Risk Manag 6: 297-305.

21. Ministère de la santé (2012) Analyse des goulots d'étranglement et de l'équité dans l'offre et l'utilisation des services de PTME. PNLS-IST du Togo, Lomé.

22. Sheahan A, Feinstein L, Dube Q, Edmonds A, Chirambo CM, et al. (2017) Early antiretroviral therapy initiation and mortality among infants diagnosed with HIV in the first 12 weeks of life: Experiences from Kinshasa, DR Congo and Blantyre, Malawi. Pediatr Infect Dis J.

23. Gamell A, Glass TR, Luwanda LB, Mapesi H, Samson L, et al. (2016) Implementation and operational research: An integrated and comprehensive service delivery model to improve pediatric and maternal HIV care in rural Africa. J Acquir Immune Defic Syndr 73: e67-e75.

24. Diallo K, Kim AA, Lecher S, Ellenberger D, Beard RS, et al. (2016) Early diagnosis of HIV infection in infants - One Caribbean and six sub-Saharan African countries, 2011-2015. MMWR Morb Mortal Wkly Rep 65: 1285-1290.

25. Celletti F, Sherman G, Mazanderani AH (2017) Early infant diagnosis of HIV: Review of current and innovative practices. Curr Opin HIV AIDS 12: 112-116.

26. Izudi J, Akot A, Kisitu GP, Amuge P, Kekitiinwa A (2016) Quality improvement interventions for early HIV infant diagnosis in north-eastern Uganda. Biomed Res Int 2016: 5625364 